\title{
Whole-genome amplification in double-digest RADseq results in adequate libraries but fewer sequenced loci
}

\author{
Bruno A S de Medeiros ${ }^{\text {Corresp., }}{ }^{1}$, Brian D Farrell ${ }^{1}$ \\ ${ }^{1}$ Department of Organismic \& Evolutionary Biology and Museum of Comparative Zoology, Harvard University, Cambridge, MA, USA \\ Corresponding Author: Bruno A S de Medeiros \\ Email address: souzademedeiros@fas.harvard.edu
}

Whole-genome amplification by multiple displacement amplification (MDA) is a promising technique to enable the use of samples with only limited amount of DNA for the construction of RAD-seq libraries. Previous work has shown that, when the amount of DNA used in the MDA reaction is large, double-digest RAD-seq (ddRAD) libraries prepared with amplified genomic DNA result in data that are indistinguishable from libraries prepared directly from genomic DNA. Based on this observation, here we evaluate the quality of ddRAD libraries prepared from MDA-amplified genomic DNA when the amount of input genomic DNA and the coverage obtained for samples is variable. By simultaneously preparing libraries for five species of weevils (Coleoptera, Curculionidae), we also evaluate the likelihood that potential contaminants will be encountered in the assembled dataset. Overall, our results indicate that MDA may not be able to rescue all samples with small amounts of DNA, but it does produce ddRAD libraries adequate for studies of phylogeography and population genetics even when conditions are not optimal. We find that MDA makes it harder to predict the number of loci that will be obtained for a given sequencing effort, with some samples behaving like traditional libraries and others yielding fewer loci than expected. This seems to be caused both by stochastic and deterministic effects during amplification. Further, the reduction in loci is stronger in libraries with lower amounts of template DNA for the MDA reaction. Even though a few samples exhibit substantial levels of contamination in raw reads, the effect is very small in the final dataset, suggesting that filters imposed during dataset assembly are important in removing contamination. Importantly, samples with strong signs of contamination and biases in heterozygosity were also those with fewer loci shared in the final dataset, suggesting that stringent filtering of samples with significant amounts of missing data is important when assembling data derived from MDA-amplified genomic DNA. Overall, we find that the combination of MDA and ddRAD results in high-quality datasets for population genetics as long as the sequence data is properly filtered during assembly. 


\section{Whole-genome amplification in double-digest RAD-seq results 2 in adequate libraries but fewer sequenced loci}

3

4 Bruno A S de Medeiros ${ }^{1}$, Brian D Farrell ${ }^{1}$

$5 \quad{ }^{1}$ Department of Organismic \& Evolutionary Biology and Museum of Comparative Zoology, Harvard

6 University, Cambridge, MA, USA

7

8 Corresponding Author:

9 Bruno A S de Medeiros ${ }^{1}$

1026 Oxford St, Cambridge, MA, 02138, USA

11 Email address: souzademedeiros@fas.harvard.edu 


\section{Abstract}

14 Whole-genome amplification by multiple displacement amplification (MDA) is a promising

15 technique to enable the use of samples with only limited amount of DNA for the construction of 16 RAD-seq libraries. Previous work has shown that, when the amount of DNA used in the MDA 17 reaction is large, double-digest RAD-seq (ddRAD) libraries prepared with amplified genomic 18 DNA result in data that are indistinguishable from libraries prepared directly from genomic DNA. Based on this observation, here we evaluate the quality of ddRAD libraries prepared from MDA-amplified genomic DNA when the amount of input genomic DNA and the coverage obtained for samples is variable. By simultaneously preparing libraries for five species of weevils (Coleoptera, Curculionidae), we also evaluate the likelihood that potential contaminants will be encountered in the assembled dataset. Overall, our results indicate that MDA may not be able to rescue all samples with small amounts of DNA, but it does produce ddRAD libraries adequate for studies of phylogeography and population genetics even when conditions are not optimal. We find that MDA makes it harder to predict the number of loci that will be obtained for a given sequencing effort, with some samples behaving like traditional libraries and others yielding fewer loci than expected. This seems to be caused both by stochastic and deterministic effects during amplification. Further, the reduction in loci is stronger in libraries with lower amounts of template DNA for the MDA reaction. Even though a few samples exhibit substantial levels of contamination in raw reads, the effect is very small in the final dataset, suggesting that filters imposed during dataset assembly are important in removing contamination. Importantly, samples with strong signs of contamination and biases in heterozygosity were also those with fewer loci shared in the final dataset, suggesting that stringent filtering of samples with significant amounts of missing data is important when assembling data derived from MDA-amplified genomic DNA. Overall, we find that the combination of MDA and ddRAD results in high-quality datasets for population genetics as long as the sequence data is properly filtered during assembly . 
40

41

42 Double-digest RAD sequencing (ddRAD) (Peterson et al., 2012) and other methods of

Introduction genotyping-by-sequencing are inexpensive and flexible tools that allow researchers to sequence

a large number of loci from non-model organisms for phylogenetic and population-level studies (Andrews et al., 2016). In spite of its many advantages, a potential barrier to the use of ddRAD is the relatively large amount of starting DNA typically required in library preparation. This may hinder its application for DNA obtained from museum specimens, rare species (Blair, Campbell \& Yoder, 2015), or small organisms (Shortt et al., 2017; Boyle et al., 2018), which yield limited amounts of DNA. A possible solution consists in preparing RAD libraries from pools of individuals, which together yield sufficient DNA (Emerson et al., 2010; Toonen et al., 2013). Pooling offers the additional benefit of reducing library preparation costs (Toonen et al., 2013; Schlötterer et al., 2014). However, accurate genotyping of pools is challenging (Cutler \& Jensen, 2010; Gautier et al., 2013; Lynch et al., 2014) and might require impractically high number of samples pooled at equimolar concentrations (Cutler \& Jensen, 2010; Lynch et al., 2014). An alternative to pooling is to prepare libraries with methods that require smaller DNA input. One of these alternatives is HyRAD (Suchan et al., 2016; Linck et al., 2017), the hybrid enrichment of genomic libraries for RAD-derived loci. One caveat is that this protocol is still not highly optimized, requiring a very large read depth in comparison to ddRAD and thus resulting in a much larger cost per sample in addition to the higher cost for library preparation. Another option for samples with smaller DNA amounts is to increase the DNA available per individual by using whole-genome amplification prior to RAD library preparation. In the context of ddRAD, Blair, Campbell \& Yoder (2015) sequenced four samples of a single species at high coverage and using a high amount of starting DNA, following the manufacturer's protocol for the reaction of whole-genome amplification. Even though their results were encouraging, it remains to be shown whether whole-genome amplification is also robust in more typical conditions in which it might be used: the study of many samples with uneven coverage and small quantities of DNA available. 
68 The method of whole-genome amplification used in this previous study, multiple displacement amplification (MDA) (Dean et al., 2002; Hosono et al., 2003), is arguably one of the best among those available in terms of detectable biases in the amplified product (Sabina \& Leamon, 2015). MDA consists of isothermal amplification of DNA using random primers for an extended time. For the applications of whole-genome sequencing and PCR, MDA has exhibited no genotyping bias and even coverage of different genomic regions under most conditions, although some studies have reported a GC-dependent coverage bias (Sabina \& Leamon, 2015). When applied to ddRAD libraries, MDA resulted in accurate genotype calls, with no detectable coverage bias (Blair, Campbell \& Yoder, 2015). These results were based on a few samples sequenced to high depth with a fairly large amount of template DNA (>100 ng). Typical population-level studies in which MDA could be most useful would include those in organisms with significantly smaller DNA yields, and possibly pools of many samples with uneven coverage. For example, Boyle et al (2018) have used MDA in ants to obtain enough DNA (150 ng) to start ddRAD library preparation from an initial template with less than $15 \mathrm{ng}$ of DNA (J. Boyle, personal communication). With even smaller extraction yields, Shortt et al. (2017) have used MDA to prepare libraries from miracidia larvae of the flatworm Schisosoma japonicum, which typically yield 1-2 ng of genomic DNA.

An important concern about whole-genome amplification, not addressed in previous studies focusing on its application to ddRAD, is nontemplated amplification. MDA reactions are known to amplify contaminants present in the MDA reagents (Sabina \& Leamon, 2015), and extraneous DNA present in the genomic DNA or the reaction setup might also be amplified and detectable in the final product. Even though any kind of ddRAD preparation starting from low amounts of DNA might be susceptible to some level of contamination, MDA may, in principle, increase the amount of contaminants in the sequenced library. While some level of nontemplated amplification can be removed by filtering out loci not shared across multiple samples during dataset assembly, the effects of nontemplated amplification on the final dataset have not been tested so far. Insects are one group of small animals for which MDA might be useful. Some small insects yield little DNA from extractions, and in these cases destructive extraction from whole bodies is 
97 needed. In our experience working with small beetles, even extractions from whole bodies 98 result in yields from a few nanograms to a few micrograms of DNA. Since the lower end of 99 these yields is also in the lower end of the input DNA required for the preparation of RAD 100 libraries (Andrews et al., 2016), MDA may enable the use of samples that yield little DNA. Here 101 we study the effects of MDA on ddRAD libraries prepared for a number of species of small (ca. $1023 \mathrm{~mm}-7 \mathrm{~mm}$ in length) weevils (Insecta, Coleoptera, Curculionidae). Taking advantage of the 103 natural variation in DNA yield across samples, we evaluate the effect of varying amounts of 104 input genomic DNA into an MDA reaction. By comparing libraries generated with and without 105 MDA from the same genomic DNA, we also evaluate the level of nontemplated amplification, 106 and the effect of MDA on heterozygosity, genotyping bias and on the loci obtained for a given 107 sequencing effort. We also test the use of a mix of amplified and non-amplified samples in 108 common population genetics analyses: population clustering and isolation by distance.

Materials \& Methods

111

\section{Sampling, library preparation and sequencing}

113 Here we work with five taxa of weevils associated with the flowers of the palms Syagrus

114 coronata and Syagrus botryophora: Anchylorhynchus trapezicollis, Andranthobius bondari,

115 Celetes impar, Microstrates bondari and Microstrates ypsilon. Our preliminary analyses using a

116 larger dataset showed evidence for cryptic speciation in Anchylorhynchus trapezicollis and

117 Andranthobius bondari. Here we will refer to these taxa here using only the generic name, but

118 we still include all samples assigned to these species based on morphology alone. Weevils were 119 collected throughout the range of their host palms between 2013 and 2014 by bagging 120 inflorescences in the staminate phase. Insects were killed and preserved in 96\% ethanol and 121 kept in a cooler and then a freezer prior to DNA extraction. All samples studied here were 122 collected with SISBIO permit number 39704-7 from the Instituto Chico Mendes de Preservação 123 da Biodiversidade, Brazil, and deposited in the Museum of Zoology of the University of São

124 Paulo. Some individuals in each species had ddRAD libraries prepared both with and without an 125 MDA step (here referred as MDA and gDNA libraries, respectively), and we also included in this 
126 study individuals with only one library prepared, either with or without MDA, that were 127 collected in the same localities as the individuals with 2 libraries. This resulted in 69 samples 128 with the 2 kinds of library prepared, 76 samples with a gDNA library only and 22 samples with 129 an MDA library only. Sample data are listed in Table S1 and a graphical overview of the samples 130 included in this study is given in Fig. 1.

131 For gDNA libraries, we have followed the protocol outlined by Peterson et al. (2012), but using 132 MagNA beads (Rohland \& Reich, 2012) instead of Ampure XP beads (Beckman Coulter) for 133 cleanup steps. DNA was extracted either automatically in an AutoGenprep 965 (AutoGen) or 134 using the EZNA Insect DNA Kit (Omega Biotek). We extracted DNA from whole bodies separated 135 between the elytra and pronotum, to preserve the remaining sclerotized tissue (the head, 136 prothorax and appendages) as vouchers, and digests were done overnight prior to extraction.

137 DNA was quantified using a Quant-iT dsDNA HS Assay Kit (ThermoFisher) in a Spectramax i3 138 plate reader (Molecular Devices). 150-300 ng of DNA was digested using the restriction 139 enzymes EcoRI-HF and Bfal (New England Biolabs), cleaned with magnetic beads and 140 quantified. 50-100 ng of digested DNA was used in a ligation reaction to add unique 5-bp inline 141 barcodes with a T4 DNA Ligase (New England Biolabs). After adapter ligation, we prepared 142 pools of 16-48 uniquely barcoded samples, which were then cleaned with magnetic beads and 143 size-selected in the range of 264-336 bp in a Pippin Prep (Sage Science). Each pool was PCR144 amplified to increase DNA amount and to add an Illumina P5 barcode using a Phusion High145 Fidelity PCR Kit (New England Biolabs), with 4-8 separate reactions ran in parallel for each pool, 146 depending on the volume obtained after size selection. We used the following program for PCR 147 reactions: initial denaturation at 98 ㅇ $\mathrm{C}$ for $1 \mathrm{~min}, 10-12$ cycles of denaturation at $98^{\circ} \mathrm{C}$ for $10 \mathrm{~s}$, 148 annealing at $65^{\circ} \mathrm{C}$ for $30 \mathrm{~s}$ and extension at $72^{\circ} \mathrm{C}$ for $30 \mathrm{~s}$, followed by a final extension at $72^{\circ} \mathrm{C}$ 149 for 7 minutes. PCR cycles were limited to 12 to avoid amplification biases (DaCosta \& Sorenson, 150 2014). PCR products from a single pool were pooled and cleaned together using MagNA beads.

151 The size distribution of final libraries was inspected in an Agilent Bioanalyzer 2100 or Agilent 152 Tapestation 2200. Libraries were quantified using a Qubit dsDNA HS Assay Kit (Thermo Fisher) 153 in a Qubit 2.0 Fluorometer (Thermo Fisher) and pooled for sequencing considering their DNA 154 concentrations and number of samples in each pool, with the aim of achieving an equal number 
155 of reads for all samples. Libraries were sequenced and Illumina barcodes demultiplexed by the

156 Bauer Core Facility at Harvard University. Pools were spread throughout multiple sequencing

157 runs to be multiplexed with unrelated more diverse libraries (usually RNA-seq) and avoid

158 problems with base calling caused by the low diversity of restriction sites and inline barcodes.

159 The minimum read length of these runs was single-end $100 \mathrm{bp}$, and all sequences with longer

160 reads were trimmed to this length prior to assembly.

161 For MDA libraries, $1.5-2 \mathrm{uL}(6-360 \mathrm{ng}$ ) of extracted DNA was amplified for 16 hours at 30ㄷ

162 followed by 3 minutes at 65으 using a Repli-G Mini Kit (Qiagen) in 15-20 uL reactions (protocol

163 in Article S1). We have performed reactions in smaller volumes than the recommended

164 manufacturer protocol $(50 \mathrm{uL})$ because smaller reaction volumes seem to reduce biases in MDA

165 for smaller DNA quantities (Sabina \& Leamon, 2015), and also result in a reduced cost per

166 sample. Following amplification, DNA was quantified with a Quant-It assay and directly used in

167 restriction digests, with the remaining library preparation following the gDNA protocol.

168

169 Bioinformatics

170 Sequences were demultiplexed by inline barcodes and assembled using ipyrad v. 0.6.8 (Eaton,

171 2014; Eaton \& Overcast, 2017). An overview of the steps in the ipyrad pipeline is given in Fig. 1.

172 No mismatches were allowed in barcodes during demultiplexing, and bases with a quality score

173 lower than 20 or containing adapters were trimmed from the $3^{\prime}$ end. After trimming, reads with

174 less than $35 \mathrm{bp}$ or with five or more low-quality bases were removed. Reads were clustered

175 both within and between samples at $85 \%$ identity, and only loci with coverage >= 7 were

176 retained for statistical base calling. In the ipyrad pipeline, genotypes are statistically called

177 independently for each sample for sites above the minimum coverage. Throughout this paper,

178 we use the phrase "assembled loci" to refer to this set of loci assembled independently for each

179 sample and filtered for minimum coverage. For assembly of the final dataset, we set, for each

180 species, the minimum number of samples for a locus to 4, and we will refer to these loci as "loci

181 in the final dataset." Input files for ipyrad are provided in Data S1. MDA and gDNA libraries from

182 a single sample were treated as different samples during assembly. Final datasets were

183 generated independently for each species by using the "branch" option in ipyrad. 
184

185

186

187

188

189

190

191

192

193

194

195

196

197

198

199

200

201

202

203

204

205

206

207

208

209

210

211

212

Effect of starting DNA amount on the number of reads obtained for MDA libraries

To test whether MDA impacts the number of loci obtained, we counted, for all MDA libraries (including those that yielded few loci or that do not have a corresponding gDNA library), the number of reads obtained and number of loci assembled. We modeled the effect of input genomic DNA used in the MDA reaction by using a linear mixed model with number of loci as the response variable, mass of input genomic DNA and number of reads as fixed effects, and sequencing pool and taxon as random effects affecting the intercept. We started by fitting a full model including all fixed effects and their interactions and simplified the model using the $\mathrm{R}$ function step to remove non-significant fixed effects stepwise using F-tests with significance level 0.05. We adopted this same procedure when fitting all mixed models throughout this study. Models were fit using the R packages Ime4 v. 1.1-12 (Bates et al., 2015) and ImerTest v. 2.0-33 (Kuznetsova, Brockhoff \& Christensen, 2015) in R v. 3.3.2 (R Core Team, 2016).

Comparisons between MDA and gDNA libraries for loci assembled, variation in coverage, GC content and heterozygosity

To characterize other possible effects of MDA, we compared several statistics between gDNA and MDA libraries. These comparisons were done only in a subset of the samples passing a minimum filter of number of loci shared between samples, to ensure that the calculated statistics were a reasonable representation of the whole set of genes potentially sequenced with ddRAD. The number of reads per sample in a pool were extremely variable. This variation is a direct result of the study design: the samples studied here are a subset of samples sequenced primarily for a phylogeographic study, and some of the re-sequenced samples had resulted in few reads during the first attempt. To overcome this limitation, we only compare gDNA and MDA libraries that resulted in at least 100 assembled loci and we use number of reads or number of loci as a covariate in all analyses. Moreover, we filtered additional samples based on the results from the analysis on non-templated amplification (see below). These showed that all samples with evidence for contamination are among those with fewer loci shared with other samples in the final dataset. To avoid any biases caused by contamination, 
213 and not MDA per se, we excluded the 9 MDA libraries with smallest number of loci in the final

214 dataset for the paired comparisons in this section. In total from the 67 pairs of libraries

215 prepared, 48 were retained after both filters. Table S1 and Table 1 include information about

216 which samples were included or excluded from each model.

217 Following sample filtering, we tested whether MDA libraries result in fewer loci than their gDNA

218 counterparts by fitting a linear mixed model with MDA and number of reads as fixed effects,

219 and sample within taxon as random effects (these same random effects were used in all mixed

220 models comparing gDNA and MDA libraries). In order to achieve approximate normality of

221 residuals, we logit-transformed the response variable, assuming a maximum of 30,000 loci for a

222 sample. We tested if MDA increases the variation in coverage across loci by fitting a model with

223 the standard deviation of coverage in assembled loci as a response and number of reads and

224 MDA as fixed effects.

225 We also tested the effect of MDA on heterozygosity and GC content by fitting a linear mixed

226 model with the same random effects as above, and MDA and number of assembled loci as fixed

227 effects. In the case of heterozygosity, we also fitted a model with MDA and average coverage

228 per assembled locus as fixed effects. Heterozygosity was calculated as observed heterozygosity:

229 the proportion of heterozygous sites for each library in loci retained in the final dataset, after

230 base calling. In all cases, if plots of residuals vs. predicted values and quantile-quantile plots

231 showed strong deviation from normality, we log-transformed the response variable or some of

232 the fixed effects. All linear mixed models fitted and data transformations are listed in Table 1.

233

\section{Biased recovery of loci under MDA}

235 After finding that MDA results in fewer sequenced loci, we tested whether this is due to 236 consistent amplification of the same set of loci or due to stochastic effects. If deterministic

237 effects predominate, it is expected that the set of loci shared by two sample should be more 238 similar between MDA libraries or between gDNA libraries than when comparing between both

239 kinds of libraries. Libraries produced from more closely related individuals are also expected to 240 produce more a similar set of loci due to higher similarity in restriction sites, and libraries that 241 were pooled together for size selection and PCR are also expected to me more similar due to 
242 more consistent size selection. Therefore, to assess whether the set of loci produced by MDA is

243 biased, we have implemented a model that jointly infers the effect of all of these variables in

244 the pairwise dissimilarity in the set of loci recovered for samples. For each taxon, we calculated

245 the pairwise dissimilarity in the final dataset as 1 - (proportion of final loci shared for both

246 libraries), producing a pairwise dissimilarity matrix for all samples for each taxon. All libraries

247 were included in this analysis, without any filter, since even libraries that do not share any locus

248 provide relevant information. We modeled the relationship between this dissimilarity matrix as

249 a response and several predictors that we believe could also be associated with the recovery of

250 a more similar set of final loci (MDA, population (i. e. locality), size selection pool and log-

251 transformed number of loci in the final dataset for each sample) using a Multivariate Distance

252 Matrix Regression (MDMR) (Anderson, 2001; Mcardle \& Anderson, 2016) implemented in the R

253 package MDMR v. 0.5.0 (McArtor, Lubke \& Bergeman, 2016; McArtor, 2017a,b). This kind of

254 regression tests the relationship between several variables and a distance matrix, drawing

255 significance from randomization. Here significant relationships indicate that similar values of a

256 given predictor are associated with libraries having more similar sets of loci in the final dataset.

257

\section{Nontemplated amplification}

259 To assess the effect of nontemplated amplification caused by MDA, we aligned reads and loci

260 obtained for each library to a reference dataset. Since no genomes are available for the species

261 studied here or for any close relatives, we produced references from the final alignments

262 generated by ipyrad. For each taxon, we combined in a fasta file all sequences from gDNA

263 libraries found in the final dataset. We included only loci from the final alignment in the

264 reference data because the filters used in ipyrad (particularly the minimum of 4 samples in an

265 alignment) should minimize the presence of contaminants in the reference set, at the cost of

266 making it less comprehensive. These sequences were de-replicated using cd-hit (Li \& Godzik,

267 2006; Fu et al., 2012) at 95\% similarity level and the resulting sequences for all species were

268 combined in a single fasta file. To assess contamination from human DNA, we also added to this

269 file the repeat-masked Human Genome Assembly GRCh38.p10, downloaded from NCBI, with

270 repeats (i. e. lower case letters) replaced by Ns prior to concatenation. 
271 This reference fasta file was used to generate a bowtie2 (Langmead \& Salzberg, 2012) database.

272 We then aligned, for all samples with both gDNA and MDA libraries, sequences to this reference

273 at three different steps during dataset assembly: adapter-trimmed reads, all loci assembled for

274 each sample with minimum coverage of 7 , and all loci for each sample that were retained in the

275 final dataset (i. e. were shared by at least another 4 samples). All alignments were made with

276 the 'very-sensitive' preset (which is equivalent to using options -D 15 -R 2 -L 22 -i S, 1,1.15) A

277 custom bash script was used to parse the SAM files produced by bowtie2 and count the number

278 of reads and clusters aligning to each reference species, as well the number of unaligned

279 sequences. Since the reference sequences are non-comprehensive and based on gDNA libraries

280 themselves, it is expected that MDA libraries will have a smaller proportion of correctly aligned

281 sequences and a larger proportion of unaligned sequences. Due to bowtie's heuristic algorithm,

282 it is also possible that a small proportion of sequences might align to incorrect taxa both in

283 gDNA and MDA libraries, especially in the case of closely related species (for species in different

284 genera, our ddRAD datasets have almost no homologous loci). However, an increase in the

285 number of incorrect alignments under MDA is only expected in the case of significant

286 nontemplated amplification. To test whether this is observed here, we modelled the effect of

287 MDA on the number of reads/loci aligning to incorrect taxa by using the logit-transformed

288 proportion of incorrect matches as the response variable in a mixed model with MDA and log-

289 transformed number of reads/loci as fixed effects and sample nested within taxon as a random

290 effect. Since some samples exhibited no incorrect match, we added 0.001 to the proportions

291 before logit transformation. We fitted three models, each at a different step in dataset

292 assembly: one for raw reads, another for all assembled loci for each sample and another only

293 including loci in the final dataset. Fitting of linear mixed models was performed as described

294 above, and all pairs of samples sharing at least 100 loci in the final dataset were included, 295 totaling 57 pairs of libraries. The same samples were included in all three models (Table 1).

296 Contamination between samples within a species would be harder to perceive and more

297 problematic in most contexts. Even though we cannot detect it directly, it is possible to test

298 whether the effects of this form of contamination cause a significant bias. To identify samples

299 that likely have a significant amount of contamination from close relatives, we compared the 
300 overall genetic distance of SNP sites between the two libraries (gDNA and MDA) produced for

301 each sample. We used as a distance metric the average number of pairwise differences

302 between the alleles of 2 libraries at SNP sites, ignoring Ns and gaps. For each gDNA library, we

303 also obtained the distance between this library and gDNA libraries from other individuals from

304 the species, if at least 35 loci were shared. The lower filter for number of loci used here was

305 necessary to include all libraries in the comparison and, as shown in the results, libraries with

306 very few loci shared with other samples were those with most problems. With little to no

307 contamination, MDA libraries are expected to be very similar to the gDNA libraries from the

308 same sample, and more similar than gDNA libraries produced from closely related individuals.

309 Therefore, we identified problematic samples as those in which their gDNA and MDA libraries

310 have higher genetic distances between themselves than the gDNA library has to its most similar

311 conspecific gDNA library. Considering that our mixed models for contamination between taxa

312 indicated that samples with higher more loci might be less affected by contamination, we also

313 observed whether samples identified as problematic using the procedures above are among

314 those with fewer loci in the final dataset.

315

\section{Overall effect of biases caused by MDA}

317 In addition to understanding the effects of MDA on specific parameters, such as heterozygosity,

318 GC bias, and amount of contaminants, we tested whether the overall biases and errors caused

319 by MDA have the potential to overcome the natural genetic variability of the populations

320 studied here and impact a population-level study. To understand if this is the case, we first

321 excluded the 9 MDA libraries with the smallest number of loci in the final dataset, since our

322 results showed that samples with significant levels of contamination are among those with

323 large amounts of missing data. Next, we have generated, for each taxon, a matrix of average

324 pairwise genetic differences in SNP sites. Since in a few cases two libraries for the same taxon

325 did not have any loci in common, a pairwise distance could not be calculated and both libraries

326 were removed from this analysis, resulting in a total of 48 samples with 2 libraries each across 5

327 taxa. We then modeled the relationship between this distance matrix as a response and several

328 predictors (MDA, population and number of loci) using a Multivariate Distance Matrix 
329 Regression (MDMR).The aim of this analysis is to test whether biases in MDA lead to spurious 330 clustering of libraries produced from different individuals. We further checked graphically

331 whether libraries from a single sample clustered together by producing neighbor-joining trees 332 based on the matrix of genetic distances.

333 As a proof of concept that MDA produces high-quality data for studies of phylogeography, we 334 have also performed population genetics analyses. Following our own recommendations of 335 imposing stringent filters for sample and locus inclusion in the final dataset, we first filtered 336 both samples and loci to reduce the amount of missing data. In the ipyrad pipeline, this is done

337 by removing specific samples and setting a minimum number of samples per locus, and we built 338 an interactive visualization tool using the R package shiny (Chang et al., 2018) (Matrix

339 Condenser, available at https://bmedeiros.shinyapps.io/matrix condenser and

340 https://github.com/brunoasm/matrix_condenser) to identify an optimal combination of sample 341 and locus filtering to reduce missing data. We aimed to retain as many samples and loci as 342 possible for each taxon with no more than $50 \%$ missing data per sample, and only removed 343 samples based on the amount of missing loci, not in their identity or library preparation 344 method.

345 For each taxon, we generated a new branch in the ipyrad pipeline using the parameters chosen 346 in Matrix Condenser. We then exported new output files containing unlinked SNPS and 347 converted these files from phylip to genpop format using a custom python script (Data S3). We 348 did a principal component analysis (PCA) of the genetic variation and k-means clustering 349 (Jombart, Devillard \& Balloux, 2010) in the R package adegenet (Jombart, 2008; Jombart \& 350 Ahmed, 2011), visualizing whether clusters obtained were compatible with geography (i. e. 351 populations) or method of library preparation. Here we are mostly concerned with whether 352 MDA results in spurious clusters, and detailed population genetic analyses with full description 353 of the populations involved and a larger sampling throughout the range of the species will be 354 published elsewhere.

355 For Celetes impar, we further tested the effects of MDA in the estimation of $\mathrm{F}_{\mathrm{ST}}$ for studying 356 isolation by distance. Since MDA may potentially bias heteozygosity, it could also have effects 357 on $\mathrm{F}_{\mathrm{ST}}$. We chose to focus only on $\mathrm{C}$. impar for this analysis because other taxa either resulted in 
358 too few pairwise population comparisons (Andranthobius, M. bondari and M. ypsilon, Fig. 1) or

359 are more complex datasets containing cryptic species (Anchylorhynchus), which require

360 analyses beyond the scope of this paper. For $C$. impar, we estimated pairwise $F_{S T}$ between

361 populations and estimated the correlation of linearized $\mathrm{F}_{\mathrm{ST}}$ with pairwise geographical distance.

362 Significance of this correlation was tested by using a Mantel test in R package vegan (Oksanen

363 et al., 2018). We repeated the analysis including only MDA samples or only gDNA libraries, and

364 observed whether correlation differed between library preparation methods. $\mathrm{F}_{\mathrm{ST}}$ was estimated

365 using the unbiased estimator of Weir and Hill (2002) combined across loci according to Bhatia

366 et al. (2013), implemented in the R package BEDASSLE (Bradburd, Ralph \& Coop, 2013). Great

367 circle distances were calculated from geographical coordinates using the R package fossil

368 (Vavrek, 2011). All R scripts used to produce statistical results and figures in this stuare

369 provided as R markdown notebooks in Article S2 (MDMR and mixed models) and Article S3

370 (population genetics analyses). All Python and bash scripts are available in Data S2 (MDMR and

371 mixed models) and Data S3 (population genetics analyses).

372

373 Results

374 Effect of starting DNA amount on the number of loci assembled for MDA libraries.

375 The amount of input DNA in the MDA reaction has a significant effect on the number of

376 assembled loci for a library (Table 1, Fig. 2). When small amounts of input DNA are used, the

377 average number of loci obtained decreases, but this effect is highly variable: some MDA

378 libraries prepared with very little DNA are comparable to gDNA libraries, while others result in

379 very few loci even with high sequence coverage.

380

381 Comparisons between MDA and gDNA libraries for loci assembled, GC content, variation in 382 coverage and heterozygosity

383 When compared to their gDNA counterparts, MDA reduces the average number of loci

384 obtained for a library, when controlling for the number of reads, and also seems to increase the

385 variation of the outcome (Table 1, Fig. 3). Even though the coefficient for MDA alone seems to

386 suggest the opposite effect, the interaction term with number of reads results in a predicted 
387 decrease of at least $29 \%$ in the number of loci assembled for number of reads above 200,000

388 (Fig. 3). While reducing the number of loci being assembled, MDA also increases the variation in coverage between loci (Table 1, Fig. 4). There is evidence that this smaller number of assembled loci is a biased set. In three of the five taxa we found significant effects of MDA on the proportion of loci shared between libraries, but the variation explained by MDA was typically half of that explained by samples being pooled together for size selection as measured by pseudo- $\mathrm{R}^{2}$. Population identity and overall number of loci obtained for a sample also had generally greater effects (Table 2 ). For both MDA libraries and gDNA libraries, heterozygosity increases with the number of loci, with the effect being more pronounced in case of MDA (Table 1, Fig. 5A). When the average coverage of assembled loci is used as predictor, we observe a positive relationship in the case of gDNA libraries but negative in the case of MDA libraries (Table 1, Fig. 5C). For gDNA libraries, the number of assembled loci and average coverage are positively correlated (Fig. 5B), but for MDA libraries this relationship is inverted because libraries assembling an unusually low number of loci also have unusually large average coverage per locus (Fig. 5B). Together, we find that average coverage and heterozygosity generally increase with the number of reads for both gDNA and MDA libraries, but in a few cases MDA libraries result simultaneously in a low number of loci and low heterozygosity. The GC content of assembled loci seems to decrease slightly with the number of loci, but it is not affected by MDA (Table 1, Fig. 6).

\section{Nontemplated amplification}

Contamination by human DNA is negligible in both kinds of libraries, with the main source of identifiable non-template sequences matching the other beetle taxa included in this study (Fig. 7). In general, most MDA libraries show a larger share of reads and loci not matching the reference dataset than their gDNA counterparts. Given that the reference set was made from

412 loci in gDNA libraries, this is not surprising. However, the proportion of unidentifiable loci is 413 small in the final dataset (Fig. 7). When compared to their corresponding gDNA libraries, the 414 increase in the proportion of reads/loci matching to sequences of an incorrect taxon for MDA 415 libraries is generally small, with only a few samples showing a very large share of incorrect 
416 matches in reads, which diminishes in the final dataset (Fig. 7). Linear mixed models did not find 417 a significant effect of MDA in the proportion of reads matching to incorrect taxa (Table 1), but a 418 higher number of reads is significantly associated with a smaller proportion of incorrect 419 matches. In the case of assembled loci, we did not find a significant effect of either MDA or 420 number of loci (Table 1). In the final dataset, we found that MDA slightly increases the 421 proportion of loci matching to incorrect taxa, and this proportion decreases with a larger 422 number of loci (Table 1). Even though the effect is significant in the final dataset, its magnitude 423 is small and the overall proportion of incorrect matches is reduced at each bioinformatics step. 424 The most extreme outlier in raw reads is represented by an MDA library that had an excess of $42525 \%$ of reads matching to an incorrect taxon when compared to its gDNA counterpart (Fig. 7). 426 The highest increase in incorrect matches for MDA samples in loci in the final dataset, on the 427 other hand, was $1.3 \%$, and the average increase was $0.1 \%$ (Fig. 7).

428 Only three libraries exhibited potential bias caused by MDA when comparing the genetic 429 distance between libraries, with distances between gDNA libraries prepared from different 430 individuals being equal to or smaller than the gDNA and MDA libraries prepared from the same 431 sample (Fig. 8). All of these MDA libraries are among the nine (15\%) with the smallest number 432 of loci shared in the final dataset, suggesting that samples resulting in unusually low number of 433 loci are more likely to have undergone significant non-templated amplification. For all further 434 analyses, to avoid the inclusion of samples with significant amount of non-template loci in the 435 final dataset, we removed these nine MDA libraries.

437 Overall effect of biases caused by MDA.

438 None of the five taxa exhibited an effect of MDA on clustering by sequence similarity, and in all 439 cases the pseudo- $R^{2}$ associated with population assignment was much larger than that 440 associated with MDA (Table 3). Moreover, in neighbor-joining dendrograms all pairs of libraries 441 prepared from the same sample correctly cluster with each other (Figure S1). In population 442 genetics analyses including both MDA and gDNA samples filtered to reduce missing data (Table 443 S2), we observe genetic clusters that correspond to cryptic species or to geography (other taxa), 444 not to library preparation method (Fig. 9). For Anchylorhynchus, clusters correspond to cryptic 
445 species. For Andranthobius, there is little genetic structure between the three localities 446 sampled and all samples are recovered as part of the same cluster. For Celetes impar, all local

447 populations are contained in one of the genetic clusters. For Microstrates ypsilon, only two 448 clusters are found, and one of the populations has individuals in both of them. This population 449 is also geographically positioned between the other two. For Microtrates bondari, two clusters 450 are found. One includes most individuals while the other includes one MDA and one gDNA 451 sample. The population divided between two genetic clusters is also the most geographically 452 isolated one. In summary, even though patterns differ between taxa, in no case we observe 453 clustering by library preparation method. For Celetes impar, patterns of isolation by distance 454 using only MDA libraries, only gDNA libraries or both together are essentially the same (Table 4, 455 Figure S2). The correlation between geographical distance and FST is very similar in these three cases, even though not significant at the 0.05 level for gDNA alone since in the filtered dataset two populations did not have any gDNA sample (Table 4).

458

459 Discussion

460 The comparison between libraries produced from the same sample with and without MDA 461 reveals that MDA can result in high-quality libraries from samples with little DNA yield, but 462 some caveats should be observed. First, there is generally a decrease in the number of 463 assembled loci for a given number of reads (Fig. 3). This contrasts with the results of Blair, 464 Campbell \& Yoder (2015), who did not find any difference in the number of loci recovered from 465 MDA and direct libraries. This difference might be explained, at least in part, by the smaller amount of input DNA used in some samples here, since we found a significant effect of amount of input genomic DNA in an MDA reaction on the number of loci, after controlling for number of reads obtained (Fig. 2). It is likely that, when low amounts of input DNA are used, stochastic effects during genome amplification may result in uneven representation of different genomic regions, decreasing the coverage of some loci below the defined threshold (Sabina \& Leamon,

471 2015). Deterministic effects could also cause a reduction in the number of assembled loci, since 472 we found evidence for gDNA and MDA libraries being associated with different sets of loci in at 473 least some taxa. This effect, however, seems to be small when compared to the effect caused 
474 by natural genetic variation between populations and of pooling samples for size selection and 475 PCR (Table 2). We have not found biased amplification of regions with different GC content, as 476 reported for MDA in previous studies (Sabina \& Leamon, 2015). It is likely that the main driver 477 of GC biases in RAD libraries is PCR amplification (DaCosta \& Sorenson, 2014), not MDA. One 478 deterministic effect that has been reported and is consistent with our results is the differential 479 amplification of loci by size. Shortt et al. (2017) found that loci recovered in MDA-amplified 480 samples are consistently shorter than loci in non-amplified samples, even with the same size 481 selection protocol. In the absence of reference genomes, we cannot evaluate fragment size 482 here, but our results are consistent with a mixture of deterministic and stochastic effects 483 resulting in the smaller set of loci for MDA samples.

484 We have found evidence for the presence of raw reads resulting from non-templated 485 amplification in a few samples, but across all samples it does not seem to be significant. Most of the reads that do not align to the correct species do not have a clear origin, but in a few cases they seem to result from contamination from other samples (Fig. 7). MDA, therefore, might add another step in which cross-contamination can occur. Even though there is some evidence for contamination in the sequenced reads, filters imposed by bioinformatics steps seem to eliminate or reduce the problem. Even in the few cases in which there is a large number of reads resulting from contamination from other species, these seem to be largely eliminated

492 from the final dataset, probably because the randomness in sources of contamination for the 493 different samples results in no shared loci derived from contaminants. It is important to highlight that we imposed non-stringent filters for minimum coverage at a locus (7) and of number of samples sharing a locus (4) for the tests of contamination. It is likely that more stringent criteria will have an even greater effect in eliminating potential contaminants.

497 We have also observed some evidence for contamination between closely related samples (Fig. 498 8), with MDA resulting in a few libraries that are genetically dissimilar to their corresponding 499 gDNA library. These libraries all exhibit a low number of loci in the final dataset, when 500 compared to other libraries for the same taxon. At least for the cases observed here, filtering

501 libraries sharing only a few loci with others in the final dataset was sufficient to eliminate 502 problematic samples. Since exclusion of samples with high amounts of missing data is already a 
503 requirement for some commonly used methods in population-level studies, such as

504 fastSTRUCTURE (Raj, Stephens \& Pritchard, 2014), ADMIXTURE (Alexander \& Novembre, 2009)

505 and DAPC (Jombart, Devillard \& Balloux, 2010), it is likely that datasets prepared for population

506 genetic analyses based on whole-genome patterns of variation would not contain significant

507 biases.

508 Even though MDA generates reliable data for population genetics, and has been successfully

509 used to study the number of queens in ant colonies (Boyle et al., 2018), it might be problematic

510 for other applications of ddRAD. For phylogenetic studies, missing data due to indels and

511 mutations in restriction sites are expected to be common, especially among more distantly

512 related species (Rubin, Ree \& Moreau, 2012). It has even been suggested that missing data

513 should not be excluded from phylogenetic datasets produced from RADseq (Eaton et al., 2016;

514 Tripp et al., 2017) and that patterns of missing loci can be used as phylogenetic characters

515 (DaCosta \& Sorenson, 2016). This contrasts with our recommendation of excluding samples

516 with high levels of missing data, and it might be hard to distinguish library preparation artifacts

517 from biological variation in the number of loci. Another potential use of ddRAD in which MDA

518 might be inadequate are genome scans for signatures of selection. Even a very small level of

519 contamination might result in false positive outlier loci, adding to the potential problems with

520 using RAD in studies of adaptation (Catchen et al., 2017; Lowry et al., 2017a,b; McKinney et al.,

521 2017).

522 MDA seems to have some effect on genotyping, since the effect of number of loci and average 523 coverage per locus is different in MDA and gDNA libraries (Fig. 5). MDA libraries with small

524 number of assembled loci are associated with large average coverage per locus and low 525 observed heterozygosity. This indicates that, in some samples, MDA over-amplified one of the

526 alleles for some loci, resulting in few loci with large coverage and apparent homozygosity. As in

527 the case of contamination, samples yielding fewer loci in the final dataset seem to be more

528 strongly affected. Using a fairly large amount of template DNA in the MDA reactions, Blair,

529 Campbell \& Yoder (2015) did not observe loss of heterozygosity in MDA libraries compared to 530 gDNA libraries. 
531 Even though MDA does introduce small errors, as we observed in heterozygosity, and a small

532 level of contamination in a few samples, these seem to be insignificant when compared to the

533 natural genetic variation of the species that we studied, and does not seem to introduce

534 systematic bias. We did not find a significant effect of MDA on the pairwise genetic distances

535 between libraries (Table 3), and neighbor-joining trees based on the pairwise genetic distances

536 clearly recovers individuals and populations (Figure S1). Further, in tests with datasets filtered

537 to exclude samples and loci with large amounts of missing data we did not find any evidence for

538 k-means clustering or $\mathrm{F}_{\mathrm{ST}}$ being biased when MDA and gDNA libraries are used in the same

539 analysis (Fig. 9, Table 4, Figure S2).

540 An important limitation of our study is the fact that sampling and sequencing was not initially

541 designed to test the effects of MDA, but rather to study the phylogeography of the species

542 included here. A major consequence is the uneven number of reads obtained between gDNA

543 and corresponding MDA libraries, since MDA libraries were sometimes produced specifically for

544 samples for which more coverage was desired. This difference in number of reads passing filter

545 should be not be understood as a result of MDA, since MDA and gDNA samples were generally

546 sequenced separately. Rather, we considered it as an independent variable that needs to be

547 accounted for while modeling. Therefore, we addressed the problem of variation in number of

548 reads per sample by removing libraries with very few assembled loci from the dataset and by

549 explicitly including the number of reads or assembled loci as predictors in our models. Another

550 important caveat is that, since variability in the amount of input gDNA in the MDA reaction

551 came from the natural variation in samples, it is possible that the effect of decreasing DNA

552 amounts that we observed is not simply a consequence of DNA quantity. It is reasonable to

553 assume that, for a given species, samples that yielded less DNA are also likely to have lower-

554 quality DNA, and therefore the effect might be largely driven by DNA quality, not quantity. It is

555 noteworthy that a relationship between sample degradation and a lower number of sequenced

556 loci has been found for ddRAD before, even in the absence of whole-genome amplification

557 (Graham et al., 2015). Considering that previous studies obtained even genomic coverage with

558 input amounts of high-quality DNA for the MDA reaction on the order of $1 \mathrm{ng}(\mathrm{Ng}$, Roberts \&

559 Coleman, 2005), it is likely that DNA quality also plays a role here and care should be taken 
560 when using MDA for sheared samples as some museum specimens. The same correlation

561 between DNA quantity and quality is probably encountered in other studies in which MDA

562 might be useful. As a consequence, we can expect that, in general, MDA can be used to rescue

563 a sample with little DNA yield that would otherwise be unsuitable for ddRAD, with a possibility

564 that the number of loci recovered will be lower than expected for a typical sample.

565

566 Conclusion

567 We characterized the effects of MDA on ddRAD libraries by making comparisons in conditions

568 experienced in studies in which MDA is most needed. Whole-genome amplification by MDA is

569 an option for increasing the starting amount of DNA for samples that would usually not be

570 suitable for ddRAD. The main problem resulting from MDA seems to be a reduction in the

571 number of loci obtained for a given number of reads, which can increase the sequencing costs

572 in addition to the cost of MDA procedure itself. Other artifacts, such as contamination and

573 genotyping bias, are not found consistently across samples and their effects are negligible for

574 the purposes of population genetic studies after samples with an unusually low number of

575 sequenced loci are filtered. In general, our sampling resulted in data generated under

576 conditions similar to those encountered in studies sequencing a large number of samples from

577 organisms with little DNA yield, and the robustness of MDA that we find here indicates that it is

578 useful, even in non-ideal conditions, in the preparation of ddRAD libraries. When proper care is

579 taken in the assembly of datasets, MDA coupled with ddRAD offers the possibility of generating

580 high-quality libraries for genomic studies in small organisms.

581

582 Acknowledgements

583 We are thankful to Sergio A. Vanin and Sonia Casari, curators of the Museum of Zoology of the

584 University of São Paulo, and the museum staff for the assistance with specimen handling and

585 loan. We also thank the staff of the Bauer core facility at Harvard for training and assistance

586 with instruments. The computations in this paper were run on the Odyssey cluster supported

587 by the FAS Division of Science, Research Computing Group at Harvard University. This 
588 manuscript was greatly improved with comments from John D. Boyle, Tauana J. Cunha, three 589 anonymous reviewers and the handling editor.

590

591 References

592 Alexander DH., Novembre J. 2009. Fast Model-Based Estimation of Ancestry in Unrelated 593 Individuals. :1655-1664. DOI: 10.1101/gr.094052.109.vidual.

594 Anderson MJ. 2001. A new method for non parametric multivariate analysis of variance. Austral 595 ecology 26:32-46. DOI: 10.1111/j.1442-9993.2001.01070.pp.x.

596 Andrews KR., Good JM., Miller MR., Luikart G., Hohenlohe PA. 2016. Harnessing the power of 597 RADseq for ecological and evolutionary genomics. Nat Rev Genet advance on:81-92. DOI: $598 \quad 10.1038 / \mathrm{nrg} .2015 .28$.

599 Bates D., Mächler M., Bolker B., Walker S. 2015. Fitting Linear Mixed-Effects Models Using $600 \quad$ Ime4. Journal of Statistical Software 67. DOI: 10.18637/jss.v067.i01.

601 Bhatia G., Patterson N., Sankararaman S., Price AL. 2013. Estimating and interpreting FST: The 602 impact of rare variants. Genome Research 23:1514-1521. DOI: 10.1101/gr.154831.113.

603 Blair C., Campbell CR., Yoder AD. 2015. Assessing the utility of whole genome amplified DNA for 604 next-generation molecular ecology. Molecular Ecology Resources 15:1079-1090. DOI: $605 \quad$ 10.1111/1755-0998.12376.

606 Boyle JH., Martins DJ., Pelaez J., Musili PM., Kibet S., Ndung'u SK., Kenfack D., Pierce NE. 2018. 607 Polygyny does not explain the superior competitive ability of dominant ant associates in 608 the African ant-plant, Acacia (Vachellia) drepanolobium. Ecology and Evolution 8:1441609 1450. DOI: 10.1002/ece3.3752.

610 Bradburd GS., Ralph PL., Coop GM. 2013. Disentangling the effects of geographic and ecological 611 isolation on genetic differentiation. Evolution 67:3258-73. DOI: 10.1111/evo.12193.

612 Catchen JM., Hohenlohe PA., Bernatchez L., Funk WC., Andrews KR., Allendorf FW. 2017.

613 Unbroken: RADseq remains a powerful tool for understanding the genetics of adaptation 614 in natural populations. Molecular Ecology Resources 17:362-365. DOI: 10.1111/1755$615 \quad 0998.12669$.

616 Chang W., Cheng J., Allaire JJ., Xie Y., McPherson J. 2018. shiny: Web Application Framework for 
617 R.

618 Cutler DJ., Jensen JD. 2010. To pool, or not to pool? Genetics 186:41-43. DOI:

$619 \quad$ 10.1534/genetics.110.121012.

620 DaCosta JM., Sorenson MD. 2014. Amplification biases and consistent recovery of loci in a 621 double-digest RAD-seq protocol. PLOS ONE 9. DOI: 10.1371/journal.pone.0106713.

622 DaCosta JM., Sorenson MD. 2016. DdRAD-seq phylogenetics based on nucleotide, indel, and 623 presence-absence polymorphisms: Analyses of two avian genera with contrasting histories. 624 Molecular Phylogenetics and Evolution 94:122-135. DOI: 10.1016/j.ympev.2015.07.026. 625 Dean FB., Hosono S., Fang L., Wu X., Faruqi AF., Bray-Ward P., Sun Z., Zong Q., Du Y., Du J., 626 Driscoll M., Song W., Kingsmore SF., Egholm M., Lasken RS. 2002. Comprehensive human 627 genome amplification using multiple displacement amplification. Proceedings of the 628 National Academy of Sciences 99:5261-5266. DOI: 10.1073/pnas.082089499.

629 Eaton DAR. 2014. PyRAD: Assembly of de novo RADseq loci for phylogenetic analyses. 630 Bioinformatics 30:1844-1849. DOI: 10.1093/bioinformatics/btu121.

631 Eaton DAR., Overcast I. 2017. ipyrad v. 0.6.8.

632 Eaton DAR., Spriggs EL., Park B., Donoghue MJ. 2016. Misconceptions on Missing Data in RAD633 seq Phylogenetics with a Deep-scale Example from Flowering Plants. Systematic biology 634 0:syw092. DOI: 10.1093/sysbio/syw092.

635 Emerson KJ., Merz CR., Catchen JM., Hohenlohe PA., Cresko WA., Bradshaw WE., Holzapfel CM. 636 2010. Resolving postglacial phylogeography using high-throughput sequencing.

637

638 Proceedings of the National Academy of Sciences 107:16196-16200. DOI:

639 10.1073/pnas.1006538107/-

640 /DCSupplemental.www.pnas.org/cgi/doi/10.1073/pnas.1006538107.

641

Fu L., Niu B., Zhu Z., Wu S., Li W. 2012. CD-HIT: Accelerated for clustering the next-generation 642 sequencing data. Bioinformatics 28:3150-3152. DOI: 10.1093/bioinformatics/bts565.

Gautier M., Foucaud J., Gharbi K., Cézard T., Galan M., Loiseau A., Thomson M., Pudlo P., 644 645 Kerdelhué C., Estoup A. 2013. Estimation of population allele frequencies from nextgeneration sequencing data: Pool-versus individual-based genotyping. Molecular Ecology 22:3766-3779. DOI: 10.1111/mec.12360. 
646 Graham CF., Glenn TC., Mcarthur AG., Boreham DR., Kieran T., Lance S., Manzon RG., Martino 647 JA., Pierson T., Rogers SM., Wilson JY., Somers CM. 2015. Impacts of degraded DNA on 648 restriction enzyme associated DNA sequencing (RADSeq). Molecular Ecology Resources 649 15:1304-1315. DOI: 10.1111/1755-0998.12404.

650 Hosono S., Faruqi AF., Dean FB., Du Y., Sun Z., Wu X., Du J., Kingsmore SF., Egholm M., Lasken 651 RS. 2003. Unbiased whole-genome amplification directly from clinical samples. Genome 652 Research 13:954-964. DOI: 10.1101/gr.816903.

653 Jombart T. 2008. Adegenet: A R package for the multivariate analysis of genetic markers. 654 Bioinformatics 24:1403-1405. DOI: 10.1093/bioinformatics/btn129.

655 Jombart T., Ahmed I. 2011. adegenet 1.3-1: New tools for the analysis of genome-wide SNP 656 data. Bioinformatics 27:3070-3071. DOI: 10.1093/bioinformatics/btr521.

657 Jombart T., Devillard S., Balloux F. 2010. Discriminant analysis of principal components: A new 658 method for the analysis of genetically structured populations. BMC Genetics 11:94. DOI: 659 10.1186/1471-2156-11-94.

660 Kuznetsova A., Brockhoff PB., Christensen RHB. 2015. Tests in Linear Mixed Effects Models. 661 Langmead B., Salzberg SL. 2012. Fast gapped-read alignment with Bowtie 2. Nat Methods 662 9:357-359. DOI: 10.1038/nmeth.1923.

663 Li W., Godzik A. 2006. Cd-hit: A fast program for clustering and comparing large sets of protein 664 or nucleotide sequences. Bioinformatics 22:1658-1659. DOI:

$665 \quad$ 10.1093/bioinformatics/btl158.

666 Linck EB., Hanna ZR., Sellas A., Dumbacher JP. 2017. Evaluating hybridization capture with RAD 667 probes as a tool for museum genomics with historical bird specimens. Ecology and $668 \quad$ Evolution 7:4755-4767. DOI: 10.1002/ece3.3065.

669 Lowry DB., Hoban S., Kelley JL., Lotterhos KE., Reed LK., Antolin MF., Storfer A. 2017a. Breaking 670 RAD: an evaluation of the utility of restriction site-associated DNA sequencing for genome 671 scans of adaptation. Molecular ecology resources 17:142-152. DOI: 10.1111/17556720998.12635.

673 Lowry DB., Hoban S., Kelley JL., Lotterhos KE., Reed LK., Antolin MF., Storfer A. 2017 b.

674 Responsible RAD: Striving for best practices in population genomic studies of adaptation. 
675

676

677

678

679

680

681

682

683

684

685

686

687

688

689

690

691

692

693

694

695

696

697

698

699

700

701

702

703

Molecular Ecology Resources 17:366-369. DOI: 10.1111/1755-0998.12677.

Lynch M., Bost D., Wilson S., Maruki T., Harrison S. 2014. Population-genetic inference from pooled-sequencing data. Genome Biology and Evolution 6:1210-1218. DOI:

$$
\text { 10.1093/gbe/evu085. }
$$

Mcardle BH., Anderson MJ. 2016. Fitting Multivariate Models to Community Data : A Comment on Distance-Based Redundancy Analysis Author ( s ): Brian H . McArdle and Marti J . Anderson Published by : Wiley Stable URL : http://www.jstor.org/stable/2680104 REFERENCES Linked references are a. Ecology 82:290-297.

McArtor DB. 2017a. MDMR: Multivariate Distance Matrix Regression.

McArtor DB. 2017b. Extending a Distance-Based Approach to Multivariate Multiple Regression. University of Notre Dame.

McArtor DB., Lubke GH., Bergeman CS. 2016. Extending multivariate distance matrix regression with an effect size measure and the asymptotic null distribution of the test statistic. Psychometrika:1-26. DOI: 10.1007/s11336-016-9527-8.

McKinney GJ., Larson WA., Seeb LW., Seeb JE. 2017. RADseq provides unprecedented insights into molecular ecology and evolutionary genetics: comment on Breaking RAD by Lowry et al. (2016). Molecular Ecology Resources 17:356-361. DOI: 10.1111/1755-0998.12649.

Ng G., Roberts I., Coleman N. 2005. Evaluation of 3 methods of whole-genome amplification for subsequent metaphase comparative genomic hybridization. Diagnostic molecular pathology : the American journal of surgical pathology, part B 14:203-12. DOI: 10.1097/01.pas.0000177801.60121.05.

Oksanen J., Blanchet FG., Friendly M., Kindt R., Legendre P., McGlinn D., Minchin PR., O'Hara RB., Simpson GL., Solymos P., Stevens MHH., Szoecs E., Wagner H. 2018. vegan: Community Ecology Package.

Peterson BK., Weber JN., Kay EH., Fisher HS., Hoekstra HE. 2012. Double Digest RADseq: An Inexpensive Method for De Novo SNP Discovery and Genotyping in Model and Non-Model Species. PLoS ONE 7:e37135. DOI: 10.1371/journal.pone.0037135.

R Core Team. 2016. R: A language and environment for statistical computing.

Raj A., Stephens M., Pritchard JK. 2014. FastSTRUCTURE: Variational inference of population 
704

705

706

707

708

709

710

711

712

713

714

715

716

717

718

719

720

721

722

723

724

725

726

727

728

729

730

731

732

structure in large SNP data sets. Genetics 197:573-589. DOI:

10.1534/genetics.114.164350.

Rohland N., Reich D. 2012. Cost-effective, high-throughput DNA sequencing libraries for multiplexed target capture. Genome research 22:939-46. DOI: 10.1101/gr.128124.111.

Rubin BER., Ree RH., Moreau CS. 2012. Inferring phylogenies from RAD sequence data. PloS one 7:e33394. DOI: 10.1371/journal.pone.0033394.

Sabina J., Leamon JH. 2015. Bias in whole genome amplification: Causes and considerations. In: Whole Genome Amplification: Methods and Protocols. 15-41. DOI: 10.1007/978-1-49392990-0_2.

Schlötterer C., Tobler R., Kofler R., Nolte V. 2014. Sequencing pools of individuals-mining genome-wide polymorphism data without big funding. Nature Reviews Genetics 15:749763. DOI: $10.1038 / \mathrm{nrg} 3803$.

Shortt JA., Card DC., Schield DR., Liu Y., Zhong B., Castoe TA., Carlton EJ., Pollock DD. 2017. Whole Genome Amplification and Reduced-Representation Genome Sequencing of Schistosoma japonicum Miracidia. PLOS Neglected Tropical Diseases 11:e0005292. DOI: 10.1371/journal.pntd.0005292.

Suchan T., Pitteloud C., Gerasimova NS., Kostikova A., Schmid S., Arrigo N., Pajkovic M., Ronikier M., Alvarez N. 2016. Hybridization capture using RAD probes (hyRAD), a new tool for performing genomic analyses on collection specimens. PLOS ONE 11:1-22. DOI: 10.1371/journal.pone.0151651.

Toonen RJ., Puritz JB., Forsman ZH., Whitney JL., Fernandez-Silva I., Andrews KR., Bird CE. 2013. ezRAD: a simplified method for genomic genotyping in non-model organisms. PeerJ 1:e203. DOI: 10.7717/peerj.203.

Tripp EA., Tsai YHE., Zhuang Y., Dexter KG. 2017. RADseq dataset with 90\% missing data fully resolves recent radiation of Petalidium (Acanthaceae) in the ultra-arid deserts of Namibia. Ecology and Evolution 7:7920-7936. DOI: 10.1002/ece3.3274.

Vavrek MJ. 2011. fossil: palaeoecological and palaeogeographical analysis tools. Palaeontologia Electronica 14:1T.

Weir BS., Hill WG. 2002. Estimating F-Statistics. Annu. Rev. Genet 36:721-50. DOI: 
$733 \quad$ 10.1146/annurev.genet.36.

734 


\section{Figure 1}

Overview of samples included in the study and the ipyrad pipeline for dataset assembly.

For each taxon, dashed rectangles represent populations and each circle represent one ddRAD library. Libraries prepared from the same individual are linked by a line. Red circles represents MDA libraries and blue represents gDNA libraries. An overview of the ipyrad assembly pipeline used to generate independent datasets for each taxon is given in the lower half of the figure and arrows indicate steps from which sequences were retrieved to study the effects of MDA. The same terminology is used throughout the text to refer to sequences at these steps. Locality data for populations is available in Table S1. 


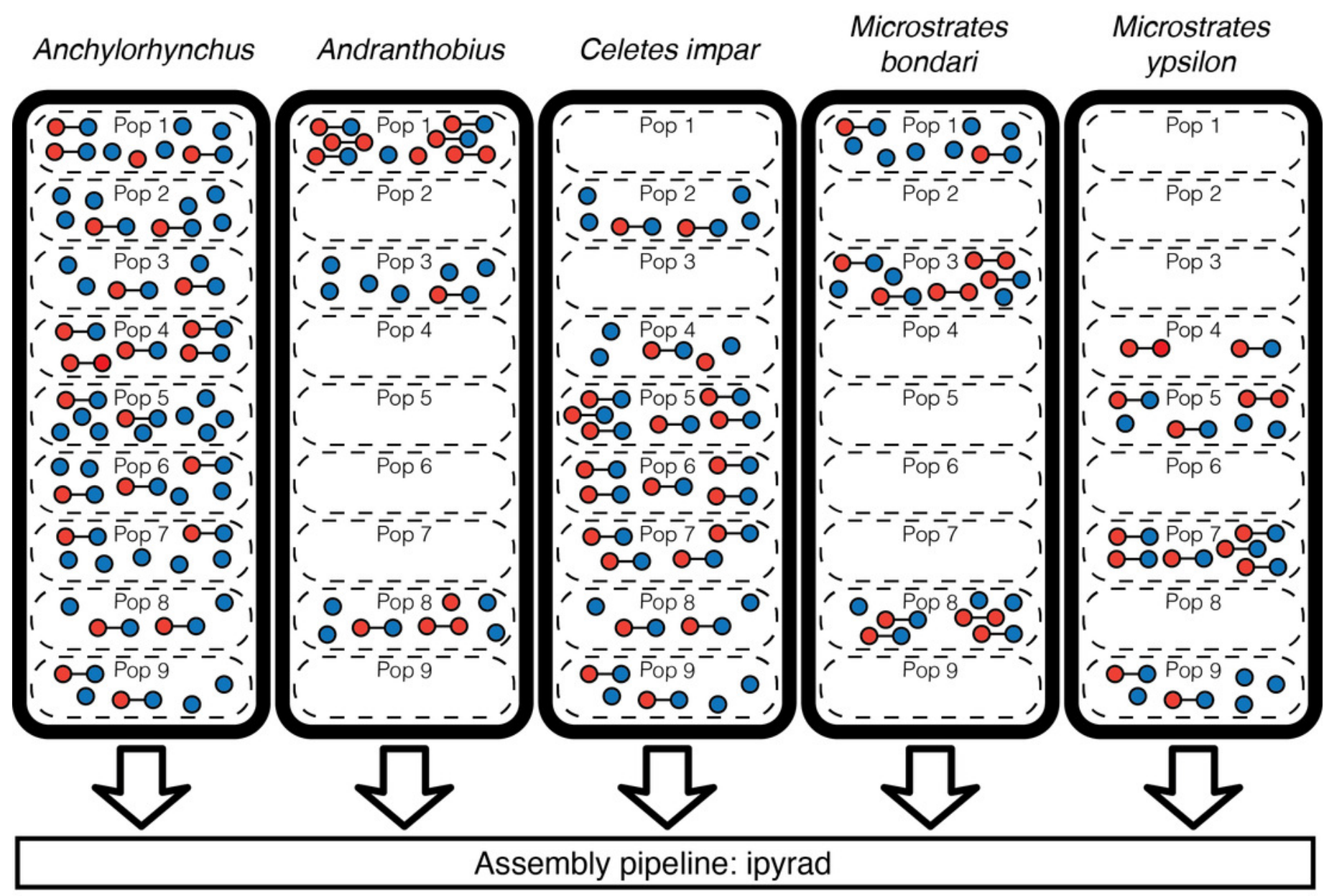

\section{约}

Step 1: Demultiplex by inline barcode

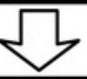

Step 2: Remove adapters, filter low-quality reads

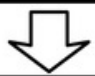

Step 3: Cluster reads within samples at $85 \%$ similarity

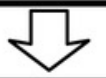

Steps 4 and 5: Filter loci with coverage smaller than 7 Statistical base calling

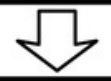

Step 6: Cluster loci between samples at $85 \%$ similarity

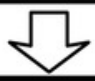

Step 7: Produce separate final datasets per taxon

Keep only loci present in at least 4 samples per taxon

Export files with final loci and called SNPs

Reads passing filter 


\section{Figure 2 (on next page)}

Small amounts of input genomic DNA in the MDA reaction reduce the number of loci assembled for a given number of raw reads.

Here we included all MDA libraries, whether or not there was a gDNA library prepared from the same sample. Colors used for both lines and points follow the same scale. The three lines represent model predictions for the relationship between number of reads and number of loci for input DNA amounts of 50 ng (brown), 100 ng (beige) and 300 ng (green). 


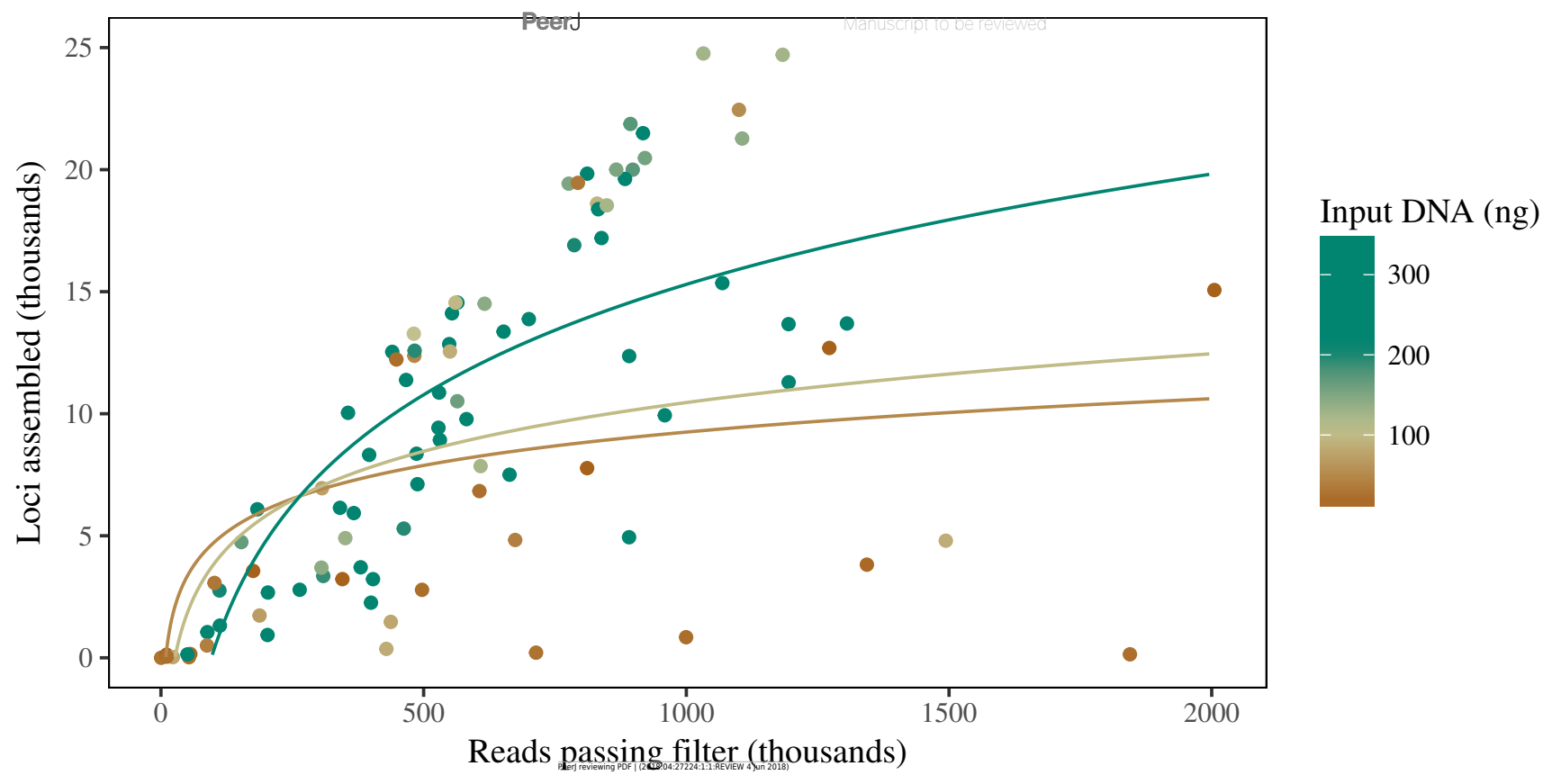




\section{Figure 3 (on next page)}

MDA reduces the number of loci assembled for a given number of reads.

Colored lines represent predictions from the mixed model for each type of library, averaging random effects. 


\section{Figure 4 (on next page)}

MDA increases variation in read depth across assembled loci.

Colored lines represent predictions from the mixed model for each type of library, averaging random effects. 


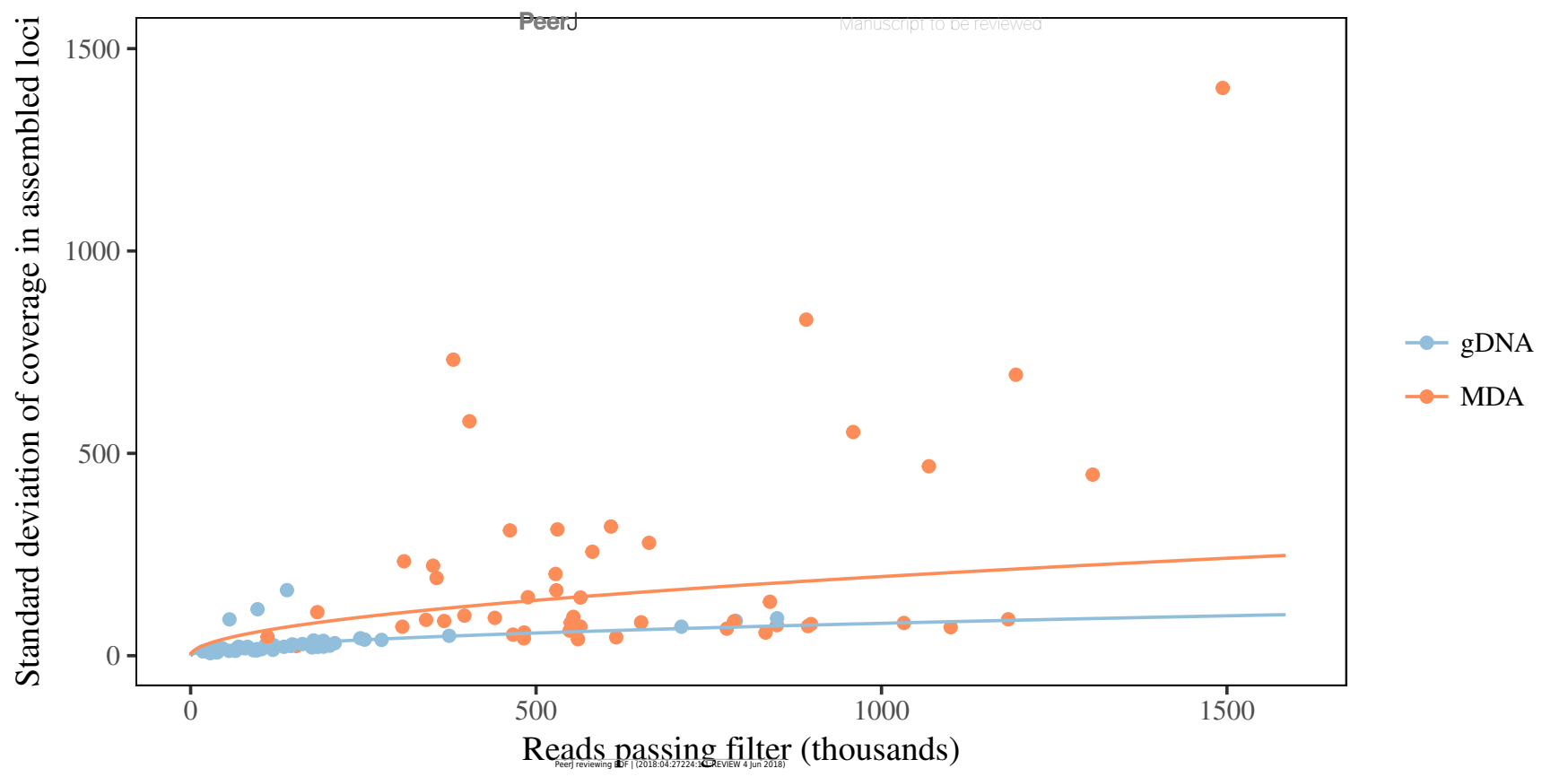




\section{Figure 5 (on next page)}

Heterozygosity depends on number of loci and average coverage, and MDA affects these relationships.

Colored lines represent predictions from the mixed model for each type of library, averaging random effects. Aheterozygosity increases with number of loci assembled, and the relationship is steeper for MDA libraries. B For gDNA libraries average coverage slightly increases with number of loci. For MDA libraries, samples with unusually low number of assembled loci have unusually large average coverage per locus. CFor gDNA libraries, heterozygosity increases with average coverage. The opposite is observed for MDA libraries. The depression in heterozygosity is more severe in samples with very high average coverage. 
Figure $\mathbf{6}$ (on next page)

MDA does not affect the GC content of loci obtained using ddRAD.

Lines represent predictions from the mixed model averaging random effects, and colors represent the kind of library. 
-PererJ

ivanuscripto be reviewea 


\section{Figure 7 (on next page)}

Effect of MDA on nontemplated amplification on reads $(A, D)$, assembled loci $(B, E)$ and loci in the final dataset (C, F).

In all graphs, all libraries are included. Only reads and loci are excluded due to filters imposed during successive bioinformatics steps (Fig. 1). For most samples, there is no detectable effect of MDA. In the few cases with large proportion of raw reads matching to the incorrect taxon, this is drastically reduced at each step in the dataset assembly. $(A, B, C)$ : Each bar represents a sample, with colors representing the proportion of reads/loci aligning to each source in the reference dataset. Correct: sequences that match to the correct taxon, Incorrect: sequences that match another weevil species, Human: sequences that match the human genome, No match: sequences that do not match any sequence in the reference dataset. Taxa are indicated in the bottom. (D, E, F): Proportion of reads/loci aligning to incorrect taxa (red in the bar graphs) against number of reads/loci sequenced, with scale of $Y$ axis constant. Arrows connect corresponding MDA and gDNA libraries. 


\section{A}

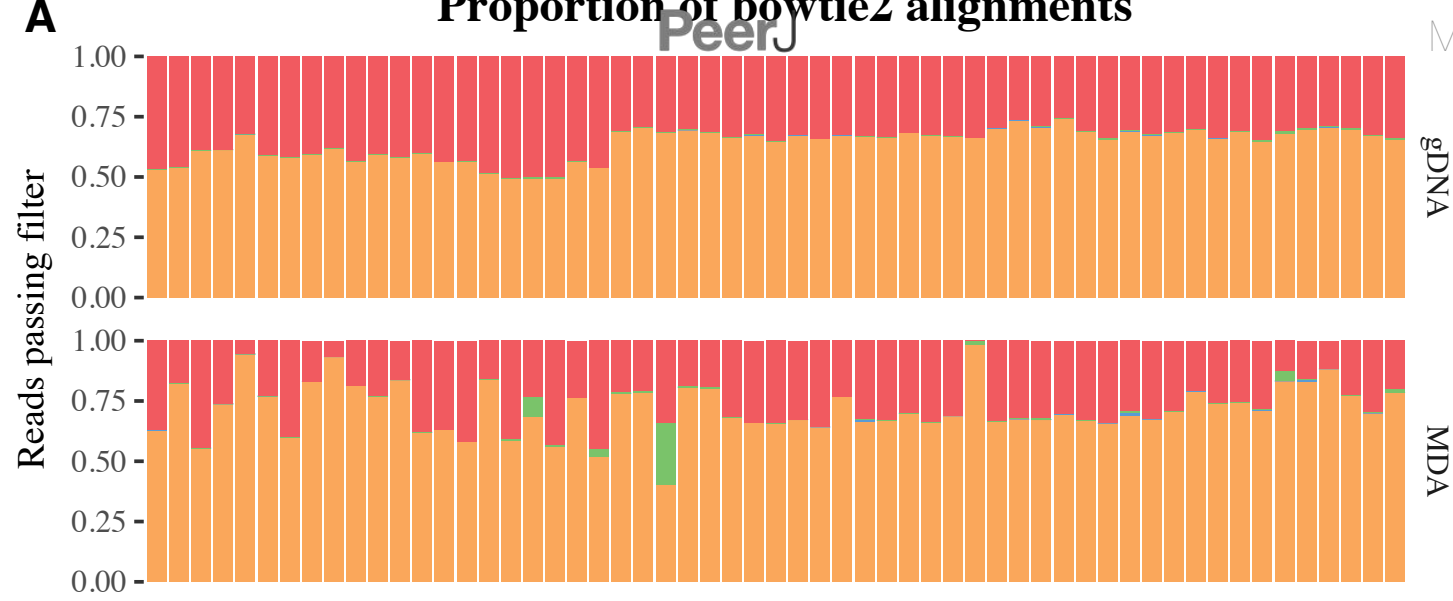

B

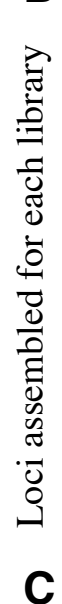

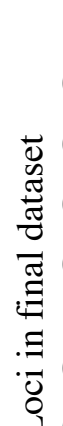

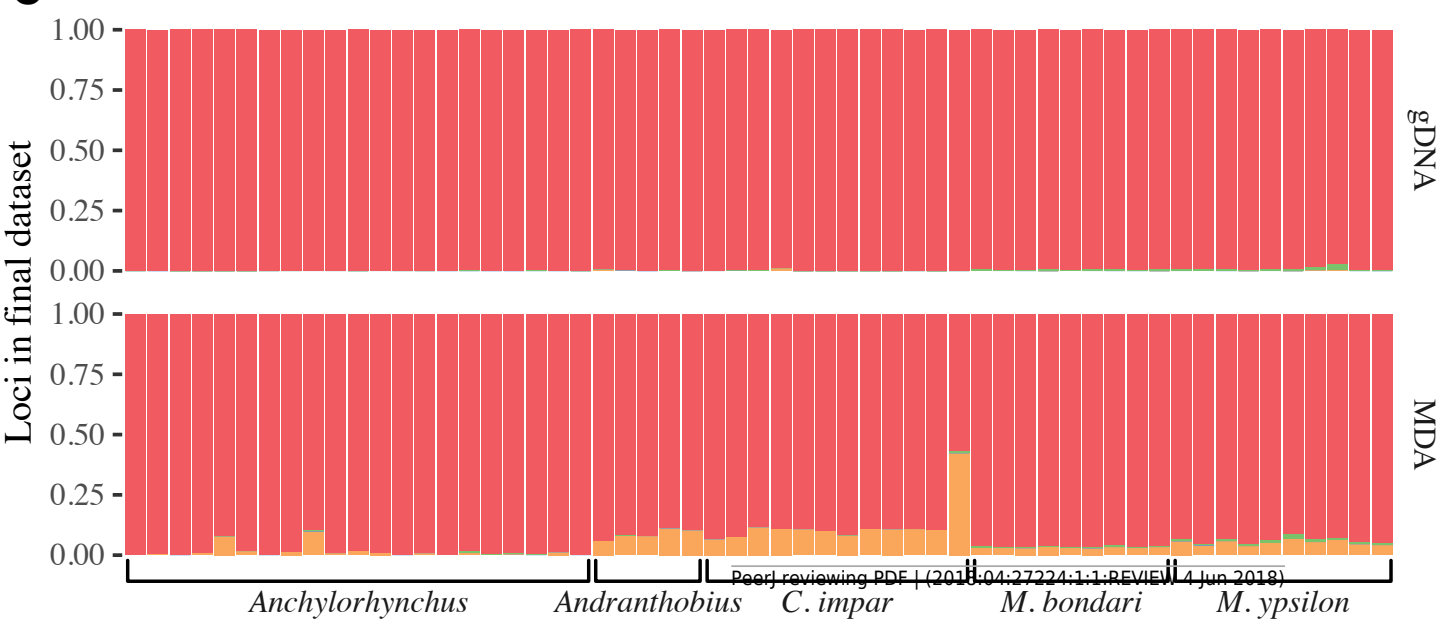

Proportion of alignments to incorrect taxa

Manuscript to
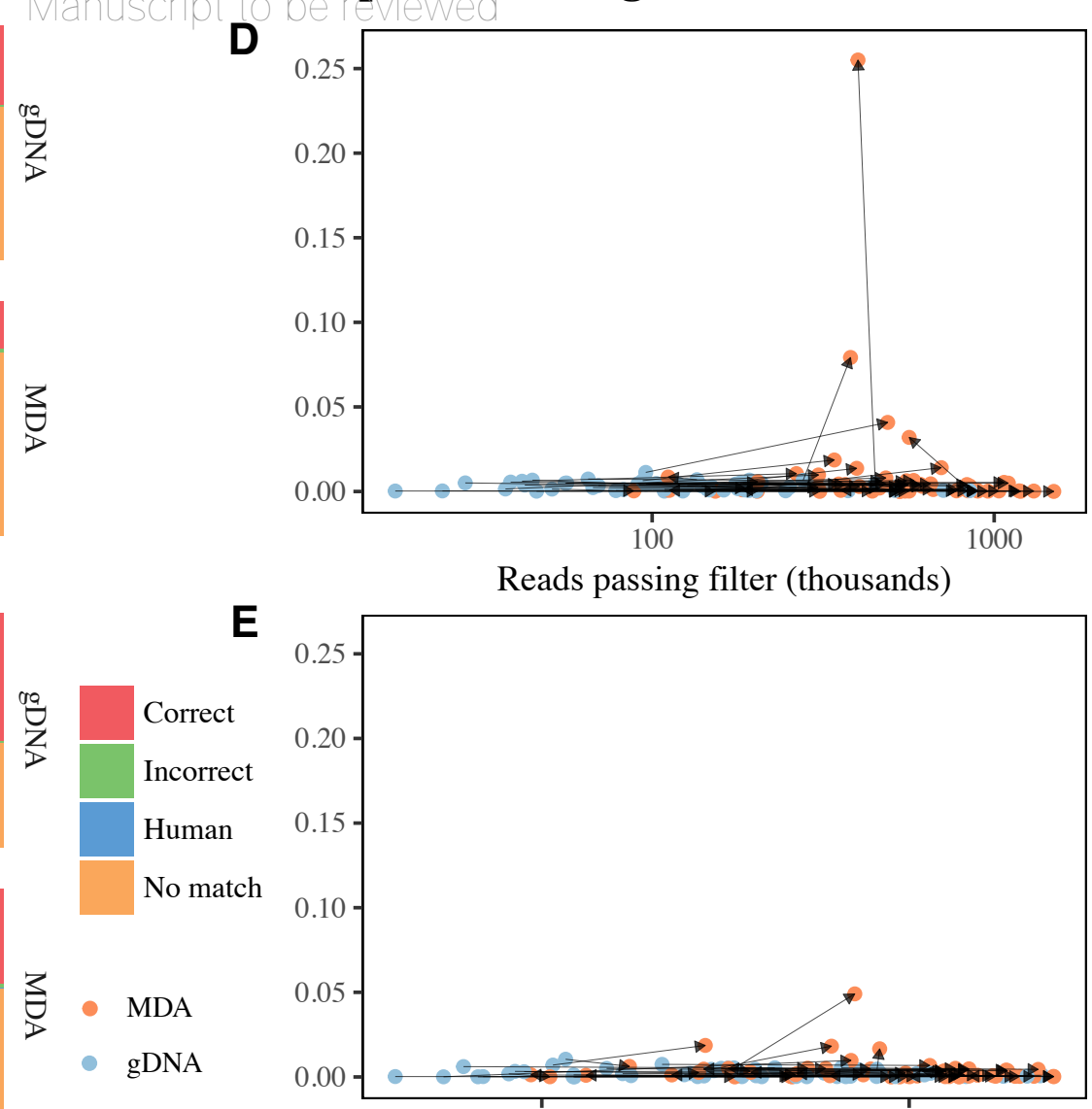

E

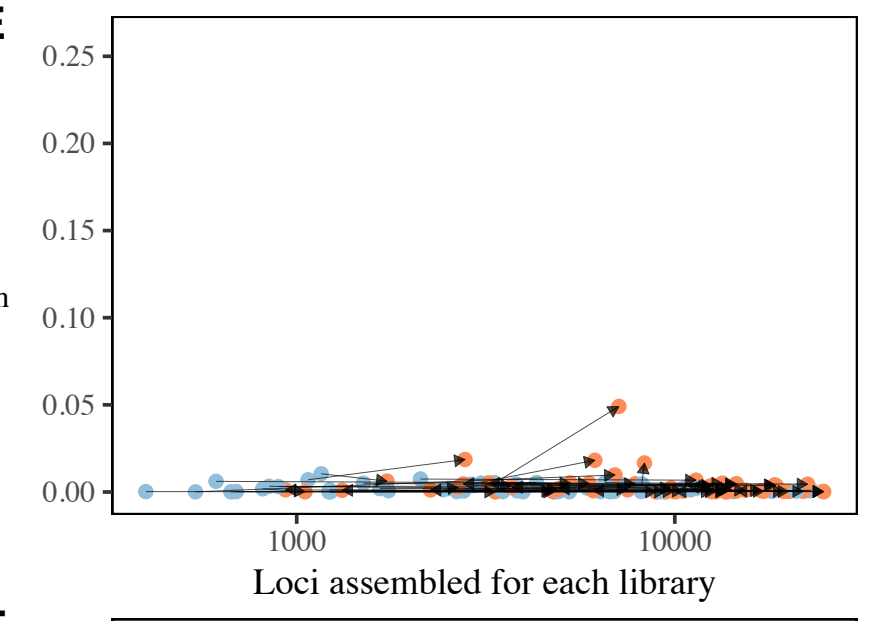

F

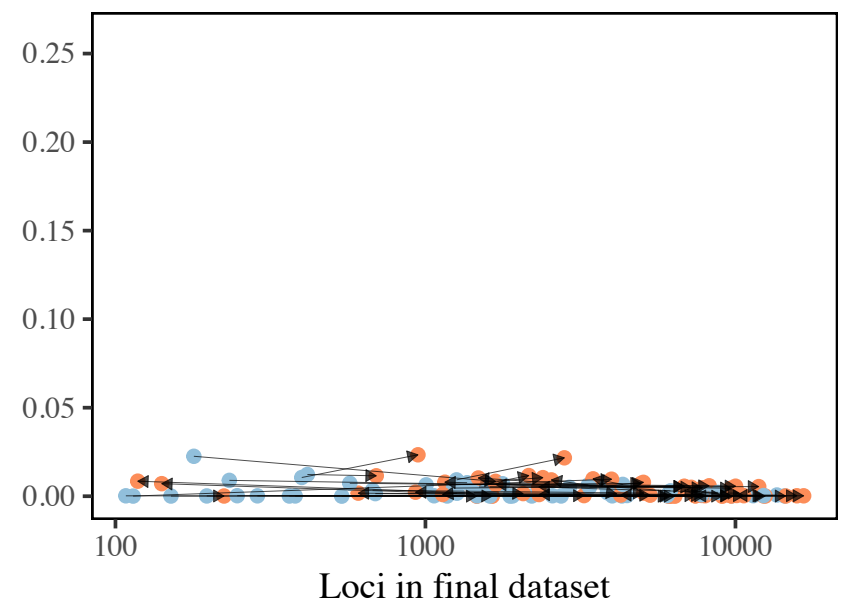


Figure 8 (on next page)

Difference between genetic distances to MDA and gDNA libraries.

For each gDNA library, values in the Y-axis are the difference between its genetic distance to its corresponding MDA library and the distance between this gDNA library and its most similar gDNA library prepared from a different sample. Values below 0 (horizontal line) indicate cases in which MDA caused enough bias as to make the genetic distance within a single individual larger than that between individuals. The three cases with evidence for such bias are among the samples with fewer loci shared in the final dataset. 
Figure 9 (on next page)

Principal component analyses and K-means clustering of weevil populations.

Dashed ellipses enclose local populations and lines connect samples that were clustered by k-means to their cluster centroid. Colors represent kind of library. Populations not shown for Anchylorhynchus (A) because this taxon consists of cryptic species largely co-distributed across several populations. 


\section{Table $\mathbf{1}$ (on next page)}

Results of mixed model fitting, with sample inclusion criteria.

Data transformations, if any, are indicated in parenthesis. Estimates and p-values are shown only for effects retained in the final models. Models in which MDA had a significant effect have their response highlighted in bold (including the first model, in which all included data came only from MDA libraries). Code and complete model summaries can be found in Article S2. 


\begin{tabular}{|c|c|c|c|}
\hline Response & Samples included & Fixed effects & Estimate (P-value) \\
\hline \multirow[t]{5}{*}{ Number of assembled loci } & All MDA libraries & Intercept & $-6624.93(0.315)$ \\
\hline & & Reads passing filter (log) & $1061.32(0.036)$ \\
\hline & & Input gDNA in MDA reaction & $-227.42(<0.001)$ \\
\hline & & (ng) & \\
\hline & & Interaction & $18.213(<0.001)$ \\
\hline \multirow{4}{*}{$\begin{array}{l}\text { Proportion of reads } \\
\text { matching to incorrect taxa } \\
\text { (logit) }\end{array}$} & 57 samples with both kinds & Intercept & $-2.83(0.034)$ \\
\hline & of library and at least 100 & Reads passing filter (log) & $-0.25(0.012)$ \\
\hline & loci in final dataset & MDA & \\
\hline & & Interaction & \\
\hline \multirow{4}{*}{$\begin{array}{l}\text { Proportion of assembled } \\
\text { loci matching to incorrect } \\
\text { taxa (logit) }\end{array}$} & 57 samples with both kinds & Intercept & $-6.30(<0.001)$ \\
\hline & of library and at least 100 & Loci with coverage >=7 (log) & \\
\hline & loci in final dataset & MDA & \\
\hline & & Interaction & \\
\hline \multirow{4}{*}{$\begin{array}{l}\text { Proportion of loci in final } \\
\text { dataset matching to } \\
\text { incorrect taxa (logit) }\end{array}$} & 57 samples with both kinds & Intercept & $-6.47(>0.001)$ \\
\hline & of library and at least 100 & Loci in final dataset (log) & $0.003(0.97)$ \\
\hline & loci in final dataset & MDA & $3.98(<0.001)$ \\
\hline & & Interaction & $-0.45(<0.001)$ \\
\hline \multirow{4}{*}{$\begin{array}{l}\text { Number of assembled loci } \\
\text { (logit) }\end{array}$} & 48 samples with both kinds & Intercept & $-19.97(<0.001)$ \\
\hline & of library and largest & Number of reads (log) & $3.55(<0.001)$ \\
\hline & number of loci in final & MDA & $4.73(0.040)$ \\
\hline & dataset & Interaction & $-0.99(0.016)$ \\
\hline \multirow{4}{*}{$\begin{array}{l}\text { Standard deviation of } \\
\text { read depth across loci }\end{array}$} & 48 samples with both kinds & Intercept & $-2.73(0.007)$ \\
\hline & of library and largest & Number of reads (log) & $1.18(<0.001)$ \\
\hline & number of loci in final & MDA & $0.89(<0.001)$ \\
\hline & dataset & Interaction & \\
\hline \multirow[t]{5}{*}{ Heterozygosity } & 48 samples with both kinds & Intercept & $-7.66 \times 10^{-4}(0.53)$ \\
\hline & of library and largest & Number of assembled loci & $5.65 \times 10^{-4}(<0.001)$ \\
\hline & number of loci in final & $(\log )$ & \\
\hline & dataset & MDA & $-7.17 \times 10^{-3}(0.012)$ \\
\hline & & Interaction & $7.69 \times 10^{-4}(0.016)$ \\
\hline \multirow[t]{4}{*}{ Heterozygosity } & 48 samples with both kinds & Intercept & $5.73 \times 10^{-4}(0.53)$ \\
\hline & of library and largest & Average coverage across & $1.09 \times 10^{-3}(<0.001)$ \\
\hline & number of loci in final & assembled loci (log) & \\
\hline & dataset & MDA & $7.99 \times 10^{-3}(0.012)$ \\
\hline
\end{tabular}




\begin{tabular}{llll}
\hline & & Interaction & $-2.21 \times 10^{-3}(0.016)$ \\
\hline \%GC & $\begin{array}{l}\text { 4 samples with both kinds } \\
\text { of library and largest }\end{array}$ & Intercept & $0.34(<0.001)$ \\
& number of loci in final & $(\mathrm{log})$ & $-1.25 \times 10^{-3}(<0.001)$ \\
dataset & MDA &
\end{tabular}

Interaction

1 


\section{Table 2 (on next page)}

Results of MDMR model fitting for dissimilarity in the set of sequenced loci for each taxon.

All paired and non-paired gDNA and MDA libraries are included. Omnibus effect is the combined effect of all predictors. Pseudo $R^{2}$ values are conceptually similar to $R^{2}$ values in a typical linear regression (McArtor, 2017b). Significant p-values are highlighted in bold.

Samples in the same pool have undergone size selection and PCR together. Population here refers to locality. 


\begin{tabular}{|c|c|c|c|}
\hline Taxon & Predictor & Pseudo $\mathbf{R}^{2}$ & P-value \\
\hline Anchylorhynchus & Omnibus effect & 0.2573 & $<0.002$ \\
\hline \multirow{4}{*}{82 libraries } & MDA & 0.0148 & 0.034 \\
\hline & Pool & 0.0547 & 0.006 \\
\hline & Population & 0.1255 & $<0.002$ \\
\hline & Loci in final dataset & 0.0214 & $<0.002$ \\
\hline Andranthobius & Omnibus effect & 0.4095 & $<0.002$ \\
\hline \multirow[t]{4}{*}{27 libraries } & MDA & 0.0478 & 0.028 \\
\hline & Pool & 0.105 & 0.002 \\
\hline & Population & 0.0701 & 0.112 \\
\hline & Loci in final dataset & 0.133 & $<0.002$ \\
\hline Celetes impar & Omnibus effect & 0.2911 & $<0.002$ \\
\hline \multirow[t]{4}{*}{43 libraries } & MDA & 0.0239 & 0.344 \\
\hline & Pool & 0.0239 & 0.346 \\
\hline & Population & 0.1214 & 0.020 \\
\hline & Loci in final dataset & 0.0794 & $<0.002$ \\
\hline Microstrates bondari & Omnibus effect & 0.2704 & $<0.002$ \\
\hline \multirow[t]{4}{*}{33 libraries } & MDA & 0.0378 & 0.06 \\
\hline & Pool & 0.062 & 0.192 \\
\hline & Population & 0.0664 & 0.054 \\
\hline & Loci in final dataset & 0.0696 & $<0.002$ \\
\hline Microstrates ypsilon & Omnibus effect & 0.2975 & $<0.002$ \\
\hline \multirow[t]{4}{*}{31 libraries } & MDA & 0.0307 & 0.608 \\
\hline & Pool & 0.0643 & 0.258 \\
\hline & Population & 0.0922 & 0.434 \\
\hline & Loci in final dataset & 0.0527 & $<0.002$ \\
\hline
\end{tabular}




\section{Table 3(on next page)}

Results of MDMR model fitting for pairwise genetic distances in each taxon.

Only paired gDNA and MDA libraries sharing loci with all samples in the final dataset are included. Omnibus effect is the combined effect of all predictors. Pseudo $R^{2}$ values are conceptually similar to $R^{2}$ values in a typical linear regression (McArtor, 2017b). Significant $p$ values are highlighted in bold. 


\begin{tabular}{llll} 
Taxon & Predictor & Pseudo $\mathbf{R}^{\mathbf{2}}$ & P-value \\
\hline Anchylorhynchus & Omnibus effect & $\mathbf{0 . 9 0 2 5}$ & $<\mathbf{0 . 0 0 2}$ \\
32 libraries & MDA & 0.0089 & 0.126 \\
& Population & $\mathbf{0 . 8 5 0 3}$ & $<\mathbf{0 . 0 0 2}$ \\
Andranthobius & Loci in final dataset & 0.0043 & 0.358 \\
6 libraries & Omnibus effect & 0.5426 & 0.664 \\
& MDA & 0.0653 & 0.890 \\
Celetes impar & Population & 0.3834 & 0.228 \\
20 libraries & Loci in final dataset & 0.0355 & 0.956 \\
& Omnibus effect & $\mathbf{0 . 6 5 0 0}$ & $<\mathbf{0 . 0 0 2}$ \\
& MDA & 0.0132 & 0.848 \\
Microstrates bondari & Omnibus effect & $\mathbf{0 . 4 8 5 0}$ & $<\mathbf{0 . 0 0 2}$ \\
18 libraries & Population & 0.6059 & 0.332 \\
& MDA & $<\mathbf{0 . 0 0 2}$ \\
& Population & $\mathbf{0 . 3 9 5 4}$ & 0.334 \\
Microstrates ypsilon & Omnibus effect & $\mathbf{0 . 5 6 2 0}$ & $<\mathbf{0 . 0 0 2}$ \\
14 libraries & MDA & 0.0328 & 0.114 \\
& Population & $\mathbf{0 . 4 0 8 9}$ & $<\mathbf{0 . 0 0 2}$ \\
& Loci in final dataset & 0.0879 & 0.710 \\
& & & $<.002$ \\
& & 0.048
\end{tabular}




\section{Table 4(on next page)}

Mantel tests for isolation by distance in Celetes impar.

See Table S2 for the number of gDNA and MDA libraries included in each population. 


\begin{tabular}{llll} 
Dataset & $\begin{array}{l}\text { Number of } \\
\text { populations }\end{array}$ & Correlation & P-value \\
\hline All samples & 6 & 0.4802 & 0.025 \\
gDNA & 4 & 0.4773 & 0.0833 \\
MDA & 6 & 0.5076 & 0.025
\end{tabular}

1 